\title{
Spatial context effects at different scales: A formal model for egocentric contexts in two- and three-dimensional space
}

\author{
Steffen Hillmert ${ }^{*}$
}

2018

DOI: 10.31235/osf.io/d4mu7

\footnotetext{
*Department of Sociology, University of Tübingen, Tübingen, Germany steffen.hillmert@uni-tuebingen.de
}

\begin{abstract}
Spatial context effects are a topic in many scientific disciplines. This paper examines which conceptual steps are necessary for determining the extension, or the appropriate scale, of relevant spatial environments when contexts can be analytically disaggregated into context elements. Adequate models of scale need to specify not only the distance-dependent relevance of context elements but also their distribution and an adequate combination. In this way, it becomes possible to understand why non-monotonic patterns in scale-related context effects have been repeatedly found in spite of common assumptions of distance decay in relevance. In many cases, a distance-related maximum of (aggregate) context relevance can be expected, so that there is an "optimal" range for the measurement of context effects. It is also important to distinguish between applications in two- and three-dimensional space. This general model helps to specify the steps necessary for the determination of spatial context patterns and to identify unrealistic assumptions.
\end{abstract}




\section{Introduction}

The role of physical space in nature and human culture is multifaceted. First, many observed findings are related to distance. This is not only reflected in formal scientific laws (e.g., Newton's law of universal gravitation [1)]) but also in more general statements such as Tobler's First Law of Geography [2]—which states that "everything is related to everything else, but near things are more related than distant things”- or the idea of distance decay $[3,4]$ (i.e., the pattern of declining relevance with distance typically found in, for example, analyses of interactions or mobility). Distance, in a general sense, abstracts from a specific metric and can be understood in various ways. Physical distance is certainly the most obvious metric, and there are many applications that use this metric, at least as a proxy, but other common metrics in specific examples (e.g., in geography) may use other metrics, including travel time (reachability) or population count (e.g., in the form of k-nearest neighbors [5]). Second, in a large number of substantive studies in various scientific disciplines such as environmental criminology, spatial epidemiology, or regional economics, to name only a few, space is associated with contextual (hierarchical) relationships. Such contextual relationships are expressed in questions such as "How is an object of study entity affected by its surroundings or by characteristics of these surroundings (context conditions)?” In fields such as (social) ecology [6-8], these questions are even among the core questions. Questions of (spatial) context effects refer to questions such as "To what degree are individual life courses affected by the level of crime in the neighborhood?" or "How is the risk of contracting a particular disease affected by the rate of infestation in the surrounding area?” or "How does pollution in the immediate environment affect the life expectancy of an organism?” Note that such questions refer to objective context effects, not context effects in the sense of contextdependent (biased) perceptions of scientific observers [9]. 
Context conditions are often measured within fixed spatial units, and they can be mapped using the spatial grid defined by these units, whereby different values of these conditions are represented, for example, by different colors (cf. Fig 1A). Contexts can, however, be constituted also by disaggregate elements that have not ex ante been assigned to defined spatial units (Fig 1B).

A

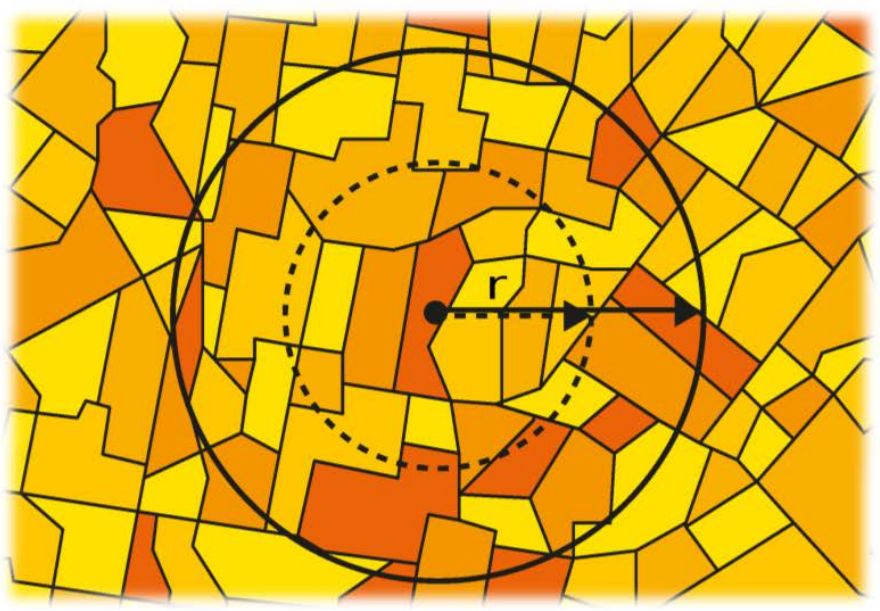

B

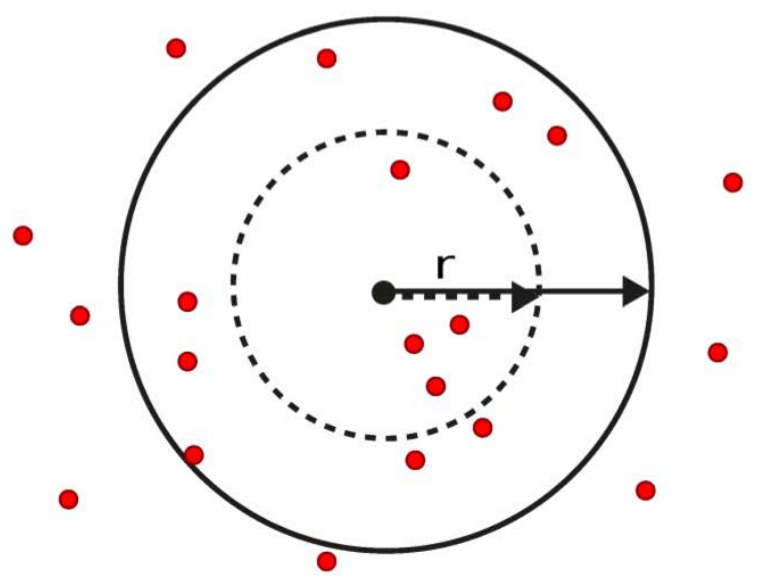

Fig 1. Context conditions in fixed spatial units and individual contexts at different scales (two-dimensional space) 
In addition, each individual object under study can be regarded to have its own specific environment. In a simplified model, we consider contexts whose frontiers are defined by a range $r$ around the individual object. This is consistent with the idea of egocentric neighborhoods or egocentric buffers or “egohoods” [10,11], where contexts are formed around an individual case. When we look at a two-dimensional space, such a context frontier is circular (Fig 1). The aspect of scaling refers to the size of the context. Applications in various scientific fields have not only shown that contexts do matter for the situation of particular cases, but also that there is a scaling problem because the measured context effects tend to vary with the scale of this context [12-18]. This means that measured effects of particular context conditions typically depend on the size of the context in which an individual case is embedded and for which the context conditions are determined. Related perspectives on this problem include the modifiable areal unit problem (MAUP), which has been discussed primarily in geography [19-21].

Beyond a lot of descriptive evidence, however, a theoretical basis for conceptualizing the scale of relevant contexts has often been lacking, and it is rather unclear how an appropriate scale for relevant contexts can be determined. There is a long tradition of creating analogies between physical and social spatial models. Probably best known are models of gravity; among others, Newton's law of physical gravitation inspired economic models of migration or international trade [22-24]. While in Newton's original formulation, gravitational force between two masses is inversely proportional to the square of the distance between their centers [1], these analogous models typically represent distance decay by a simple inverse function of distance. Gravity is used as a measure of relevance without the implication of a directed force, as in physics. Even more important, these statements and models typically refer to bilateral relationships (how one entity is affected by another entity), not to aggregate spatial contexts. Going beyond that, this article investigates what can be formally stated about 
the extension of relevant contexts apart from or in addition to issue-specific aspects. It therefore develops a framework of how to conceptualize influences in hierarchical contexts and their spatial extension. The main argument is that contexts can be analytically disaggregated into context elements and that adequate models of scale need to include not only the distance-dependent relevance of context elements (as it is expressed in notions such as distance decay) but also their number, which is also distance-dependent.

\section{A model of spatial contexts and their elements}

Drawing upon the ideas of egohoods with varying radii, spatial patterns in the relevance of the context can be described in relation to the distance to the individual case under study. Typical patterns include (A) monotonic decline of context relevance with distance or (B) a non-monotonic distance-related profile (cf. Fig 2).

A

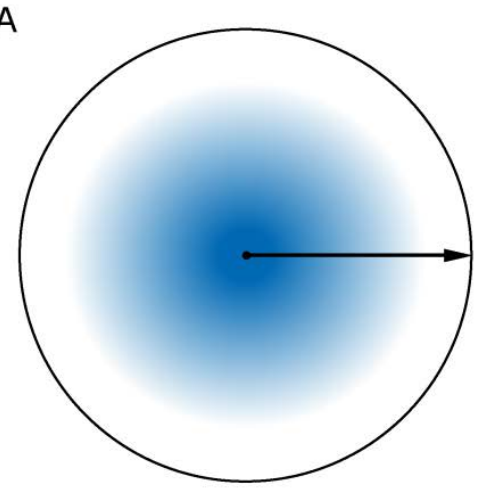

B

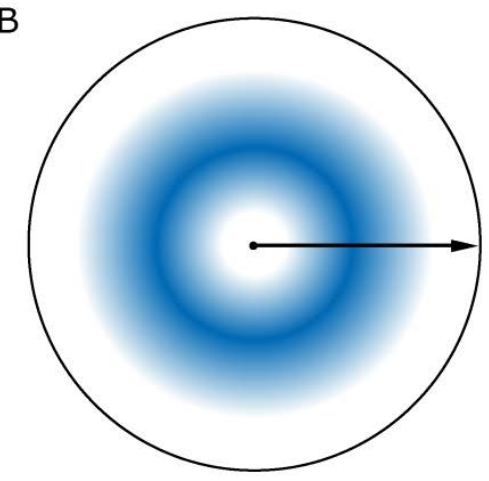

Fig 2. Typical patterns of distance-related context relevance (two-dimensional space)
(A) monotonic distance decay
(B) non-monotonic pattern (maximum in medium range)
Dark areas represent high context relevance. 
However, without further specification, illustrations of context relevance as in Fig 2 cannot be interpreted without ambiguity. This becomes clear when applying a disaggregated model of contexts. In such a theoretical model, contexts can be broken down into a combined set of context elements that are relevant for the object under study. Such elements may be jobs that can be taken up (defining "labor market conditions" as context characteristics), possible sources of infection (defining "infestation with a disease" as a context characteristic), and more; for an early concept of context elements in a geographical space, see [25]. These elements are distributed in space; they are the basis of the calculation of context conditions (e.g., the proportion of context elements that have a particular characteristic X).

Context relevance in range $\boldsymbol{r}$ can then refer to three different possible aspects that should be clearly distinguished (cf. Fig 3): (A) to a single-element (in theory, zerodimensional) context in distance $\boldsymbol{r}$; $(B)$ to a marginal (one-dimensional) context in distance $\boldsymbol{r}$ (i.e., the circle line in radius $r$ ); or $(C)$ to an area-based (two-dimensional) context within distance $\boldsymbol{r}$ (i.e., the area covered by radius $\boldsymbol{r}$ ). 
A

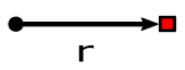

B

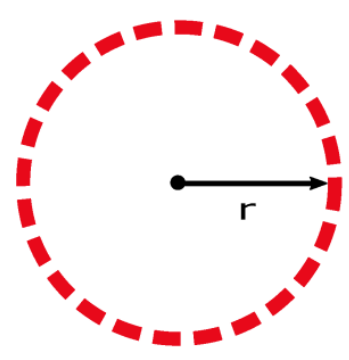

C

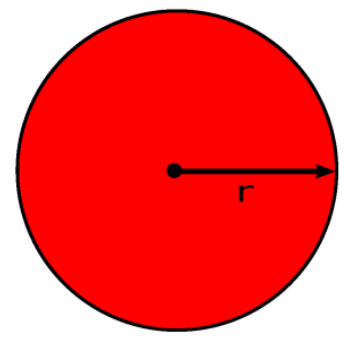

Fig 3. Context relevance in disaggregated contexts
(A) single-element context
(B) marginal context
(C) area-based context

In the following section, a formal, generic model of context relevance for the various definitions of context will be developed. This model abstracts from a particular metric of distance, although for population-based metrics, some of the specified conceptual elements may coincide or overlap. 


\section{Determining context relevance in two-dimensional space}

Problems of contexts in two-dimensional space, at least as an approximation, are frequent in academic disciplines like geography, economics, the social sciences, and biology.

Starting with an object under study and a single context element, we can imagine a potential impact of this context element. Therefore, let $\boldsymbol{a}$ be the effect on an entity of an attribute characterizing a single context element when there is no relevant distance to the object of study. Such an effect could represent, for example, the change in the probability of taking a job given a vacancy at a particular employer or the probability of contracting a disease from an infected person. This elementary effect has, by definition, no spatial dimension; inherent characteristics and intervening causal mechanisms determine how relevant a particular element is for a particular object of study.

Then, $\boldsymbol{E}(\boldsymbol{a}, \boldsymbol{n})$ is the combined effect on the object of study of this attribute in $\boldsymbol{n}$ context elements. Typically, a number of elements have a combined effect on the entity under study that is different from the effect of a single context element. This function represents a substantive, problem-specific relationship and, again, has no spatial dimension. For example, a linear relationship would mean that the combined effect from $\boldsymbol{n}$ context elements is the sum of effects from each of their effects; a function representing decreasing returns such as a root function or a log function would mean that the combined effect is smaller than the sum of all single effects.

In the following steps, we extend this non-spatial relationship by considering the distances between the entity under study and the various elements of the context. Apart from their distance to the object of study, all elements are regarded as equal. 
There is normally no clear-cut spatial distinction between relevant and irrelevant context areas. Rather, relevance will be a matter of degree, and distance will be a major aspect of this degree. We, therefore, need a probabilistic concept.

Let $\mathbf{a R}(\mathbf{r})$ be the effect of an attribute characterizing an element in a spatial distance $\boldsymbol{r}$, with $\mathbf{R}(\mathbf{r})$ the distance-related relevance of the element $(\boldsymbol{r} \geq 0)$.

Such a functional relationship between the distance of a context element to the object of study and the relevance of this element can be represented by an element-specific relevance curve. Considerable variation in the form of this function can be expected. Conventional assumptions of distance decay typically refer to such a relationship. Substantive mechanisms behind such a decline in relevance with distance include distance-based variation in the likelihood of encountering a context element and differences in the likelihood of taking up an opportunity due to distance-related costs of information, access, and mobility.

The relevance function is related to one particular context element, but the relevance of single context elements is not the only dimension that changes with distance. Within a certain radius (or "range”), the number of context elements typically increases when the size of the context where the context elements are located increases.

Therefore, let $\mathbf{N}(\mathbf{r})$ be the number of context elements in a spatial distance $\boldsymbol{r}(\boldsymbol{r} \geq 0)$ (i.e., the number of context elements on a circular line around the object of study).

$$
\boldsymbol{N}(\boldsymbol{r})=\mathbf{2} \boldsymbol{r} \boldsymbol{n}(\boldsymbol{r}) \boldsymbol{\pi} \quad \text { with } \mathrm{n}(\mathrm{r}) \text { the element density at distance } \boldsymbol{r}
$$


The relevance of context conditions can then be conceptualized as the product of (undirected) element-specific relevance and the number of context elements. Hence, this marginal relevance $\boldsymbol{M R}$ of a context, in the shape of a circular line with radius $\boldsymbol{r}$, is

$$
\operatorname{MR}(\boldsymbol{r})=R(\boldsymbol{r}) N(\boldsymbol{r})
$$

Calculating the expected relevance of conditions within a context area requires a measure of the average relevance of context elements within this area.

The number of context elements within a circular area is

$$
\int_{0}^{r} N(\rho) d \rho .
$$

Performing weighted integration across the expanding circle line and dividing by number of context elements in the circle area results in the average relevance of context elements within radius $\boldsymbol{r}$, which is

$$
\frac{\int(3)}{(4)}=\frac{\int_{0}^{r} R(\rho) N(\rho) d \rho}{\int_{0}^{r} N(\rho) d \rho}
$$

$$
\text { (4) } \times(5) \text { is the coverage of relevant context elements by range } \boldsymbol{r}
$$

This product of both the number of context elements and their average relevance as a function of radius increases and converges toward a saturation level. Still, this is not a measure of the expected context effects in an area defined by a particular radius. For the 
calculation of context variables, it needs to be taken into account that any larger context area includes all the relevant context elements, but with increasing size, these represent a smaller and smaller share of all context elements. However, context variables are calculated on the basis of all context elements in the chosen area. This means that with increasing distance, the additionally considered context elements may be less and less informative about the proximate, relevant context elements. Taking into account this increasing irrelevance, a factor representing the proportion of relevant context elements also has to be considered. The proportion of relevant context elements within range $r$ is identical to the average relevance (5). This proportion multiplied by the coverage is the relevance of a circular context with radius $\boldsymbol{r}$.

$$
\boldsymbol{C R}(\boldsymbol{r})=(5) \times(6)=(5) \times(4) \times(5)=\frac{\left(\int(3)\right)^{2}}{(4)}
$$

To get an estimate of the expected size of context effects, we need to consider the combination function $\boldsymbol{E}(\boldsymbol{a}, \boldsymbol{n})$ introduced above. This function represents the combined effect from $\boldsymbol{n}$ context elements on the object of study. Applying this function results in the expected context effects in contexts of different sizes.

Using

$\boldsymbol{E}(\boldsymbol{a}, \boldsymbol{n})$, the combination function,

the combined (context) effect from all elements at distance $\boldsymbol{r}$ (i.e., on a circular line with radius $\boldsymbol{r}$ ) is 


$$
\begin{gathered}
E(\boldsymbol{a}, \boldsymbol{r})=E(\boldsymbol{a}, M R(\boldsymbol{r})) \quad \text { with } \quad M R(\boldsymbol{r})=R(\boldsymbol{r}) N(\boldsymbol{r}) \\
\text { and } \quad N(\boldsymbol{r})=2 \boldsymbol{r} \boldsymbol{n}(\boldsymbol{r}) \pi .
\end{gathered}
$$

The combined (context) effect from all elements within distance $\boldsymbol{r}$ (i.e., in a circular area with radius $\boldsymbol{r}$ ) is

$$
E(a, r)=E(a, C R(r)) \quad \text { with } \quad C R(r)=\frac{\left[\int_{0}^{r} R(\rho) N(\rho) d \rho\right]^{2}}{\int_{0}^{r} N(\rho) d \rho}
$$

and $\quad N(r)=2 r n(r) \pi$.

\section{Determining context relevance in three-dimensional space}

The considerations of the previous section can be generalized for three-dimensional contexts. Such contexts are particularly relevant for applications involving voluminous environments (e.g., in the atmosphere or the ocean). In three-dimensional space, the surface of context elements in a given distance $\boldsymbol{r}$ is described not by a circle, but by a ball (Fig 4A), and the three-dimensional context is represented by the volume of the ball (Fig 4B). Again, various spatial patterns of context relevance are possible (cf. Fig 5). 
A

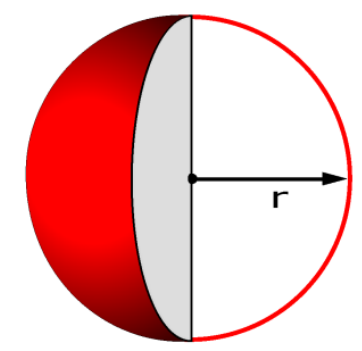

B

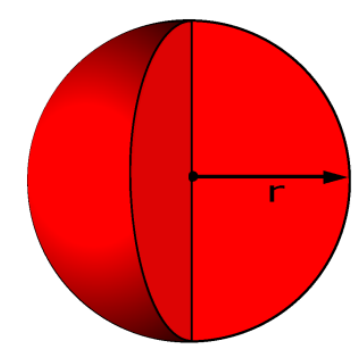

Fig 4. Contexts defined by range $r$ (three-dimensional space)
(A) marginal context
(B) volume-based context
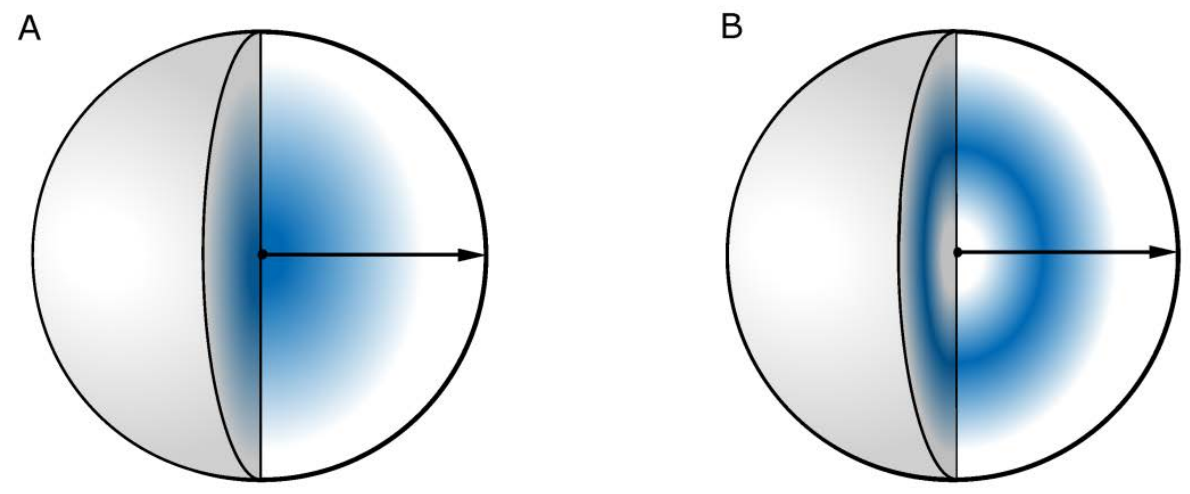

Fig 5. Typical patterns of distance-related context relevance (three-dimensional space)
(A) monotonic decay
(B) non-monotonic pattern (maximum in medium range)
Dark areas represent high context relevance. 
In analogy to the previous section,

$\mathbf{R}(\mathbf{r})$ is the distance-related relevance of the element $(\boldsymbol{r} \geq 0)$

$\mathbf{N}(\mathbf{r})$ is the number of context elements in a spatial distance $\boldsymbol{r}(\boldsymbol{r} \geq 0)$, meaning the number of context elements on a ball surface around the object of study.

$$
\boldsymbol{N}(\boldsymbol{r})=\boldsymbol{n}(\boldsymbol{r}) \mathbf{4} \boldsymbol{r}^{2} \boldsymbol{\pi}, \quad \text { with } \boldsymbol{n}(\boldsymbol{r}) \text { the element density at distance } \boldsymbol{r}
$$

The relevance of context conditions can then be conceptualized as the product of undirected element-specific relevance and the number of context elements. Hence, this marginal relevance of a context in the shape of a ball surface at radius $\boldsymbol{r}$ is

$$
\operatorname{MR}(\boldsymbol{r})=\boldsymbol{R}(\boldsymbol{r}) \boldsymbol{N}(\boldsymbol{r})
$$

The number of context elements within the ball is

$$
\int_{0}^{r} N(\rho) d \rho
$$

Weighted integration across the expanding ball surface and dividing by the number of context elements in the ball volume results in the average relevance of context elements within radius $\boldsymbol{r}$, which is

$$
\frac{\int(13)}{(14)}=\frac{\int_{0}^{r} R(\rho) N(\rho) d \rho}{\int_{0}^{r} N(\rho) d \rho}
$$


$(14) \times(15)$ is the coverage of relevant context elements by range $\boldsymbol{r}$

The proportion of relevant context elements within range $\boldsymbol{r}$ is again identical to the average relevance (15). This proportion multiplied by the coverage is the relevance of a ballshaped context with radius $\boldsymbol{r}$.

$$
C R(r)=(15) \times(16)=(15) \times(14) \times(15)=\frac{\left(\int(13)\right)^{2}}{(14)}
$$

Finally, we again consider the combination function $\boldsymbol{E}(\boldsymbol{a}, \boldsymbol{n})$, which represents the combined effect from n elements on the object of study. Applying this function results in the pattern of the expected context effects within ball-shaped contexts of a particular size.

Using

$$
\boldsymbol{E}(\boldsymbol{a}, \boldsymbol{n}) \text {, the combination function, }
$$

the combined (context) effect from all context elements at distance $\boldsymbol{r}$ (i.e., on a ball-shaped surface with radius $\boldsymbol{r}$ ) is

$$
E(\boldsymbol{a}, \boldsymbol{r})=E(\boldsymbol{a}, M R(\boldsymbol{r})) \quad \text { with } \quad \operatorname{MR}(\boldsymbol{r})=\boldsymbol{R}(\boldsymbol{r}) \boldsymbol{N}(\boldsymbol{r})
$$

$$
\text { and } \quad N(r)=n(r) 4 r^{2} \pi
$$


The combined (context) effect from all context elements within distance $\boldsymbol{r}$ (i.e., within a ball of radius $\boldsymbol{r}$ ) is

$$
E(a, r)=E(a, C R(r)) \quad \text { with } \quad C R(r)=\frac{\left[\int_{0}^{r} R(\rho) N(\rho) d \rho\right]^{2}}{\int_{0}^{r} N(\rho) d \rho}
$$

and $\quad N(r)=n(r) 4 r^{2} \pi$

\section{Selected examples}

On the basis of this model, the expected patterns of context relevance can be derived from specific assumptions about the element-specific relevance, the distribution of context elements, and the combination function. In the following, selected examples are presented. In these cases, calculations were done numerically, but a number of combinations may also allow an analytical solution. When investigating, and statistically estimating, context effects for a number of egohoods, these functions typically refer to averages, which may not represent exactly the specific situation in every egohood. Radius-based illustrations of applications in two-dimensional space are displayed, in stylized form, in Fig 6.

In example $(A)$, simple distance decay is assumed (i.e., the relevance of context elements is proportional to the inverse of distance (1)). This matches adaptations of the gravity model in the social sciences and economics [22-24,26]. Context elements are assumed to be equally distributed within the two-dimensional space (2). Following these assumptions and the formulae developed above, the marginal context relevance (3), the area-based context relevance (7), and all the intermediate steps can be calculated. Depending on the respective combination function (8), the distance-based effect pattern of both the marginal context effect (9) and the context effect within the area (10) can be derived. In models (i) and (ii), these effects are constant with distance regardless of whether a linear combination function or a 
function representing decreasing returns (square root of context relevance) is chosen. It is likely that for most applications, this result indicates that the assumption of relevance as a simple inverse function of distance is unrealistic, at least for an unlimited scale, because it would imply an equal level of (aggregate) context effects regardless of the size of the egohood and, hence, also for larger and larger context areas. Models (iii) and (iv) consider local clustering of context elements around the objects of study (agglomeration), which is a typical feature of, for example, human population patterns and corresponding representative sample data. When clustering is represented by the function $1 / \mathrm{r}$, the context effects can be expected to decline with distance.

Example $(B)$ follows the original form of the gravity model, assuming a decline of the element-specific relevance, which is proportional to $1 / \mathrm{r}^{2}$. For both an equal distribution of context elements and a central clustering, the expected patterns of context effects are a decline with distance, to varying degrees. Example (Bi) matches the respective propositions from social impact theory in psychology. Referring to the likelihood of social interactions, this theory states that the influence of others is an inverse square function of distance. Given an equal distribution of people in space, the number of interactions should then decrease as a simple inverse function of distance $\left(r \times 1 / r^{2}=1 / r\right)$ [27]. Note that this statement refers to what is termed the "marginal context” (columns (3) and (9) in Fig 6) in this article.

In example $(C)$, it is assumed that the element-specific relevance follows a normal density. This represents a profile where differences in distance do not matter much within a small range but do matter in larger ranges. Beyond a certain range, the relevance of context elements becomes eventually very small. Such a feature can be expected to apply to many aspects of human behavior, such as commuting behavior. In this case, we can also expect nonmonotonic patterns of context effects (i.e., that there is a maximum of expected context effects in an intermediate range). This means that it is often unlikely to expect marked context in very small proximate spatial contexts. While each of the respective context elements or events in 
this range may be very relevant, there are too few of them to become salient. In contrast to this, a multitude of context elements or events can be found in large-scale contexts, but most of them are too far and, hence, are not relevant enough to be salient. Model $(C)$ applies (idealtypically) to many applications in the social sciences concerning local context effects on individual behavior such as the effects of local economic circumstances on job and career decisions. Minor differences in distance within the immediate environment will not be of great importance to individuals. For example, it is probably not very important to an individual whether a job is available at a distance of three kilometers as opposed to two. However, the costs of pursuing opportunities increase progressively with distance and, at some point, become too high to be attractive or even realistic. Such a functional relationship between the distance to the individual and the relevance of an opportunity is represented by an S-shaped or half bell-shaped opportunity-specific relevance curve (for empirical confirmation of this functional type, see $[28,29])$. Model $(C)$ predicts a curvilinear pattern for the scaledependence of context effects, meaning that the context effect in an "optimal” range can be expected to be larger than within either a smaller range or a larger range. 


\begin{tabular}{|c|c|c|c|c|c|c|c|c|c|c|}
\hline & (1) & (2) & (3) & (4) & (5) & (6) & (7) & (8) & (9) & (10) \\
\hline & $\begin{array}{c}\text { Relevance } \\
\text { of opp. by } \\
\text { distance: } \\
R(r)\end{array}$ & $\begin{array}{l}\text { Number of } \\
\text { opp. by } \\
\text { distance: } \\
N(r)\end{array}$ & $\begin{array}{l}\text { Marginal } \\
\text { relevance } \\
\text { MR (on } \\
\text { circle line r) }\end{array}$ & $\begin{array}{l}\text { No. of opp } \\
\text { within circle } \\
\text { (r) }\end{array}$ & $\begin{array}{c}\text { Average } \\
\text { relevance } \\
\text { within circle } \\
\text { (r) }\end{array}$ & $\begin{array}{c}\text { Coverage } \\
\text { within circle } \\
\text { (r) }\end{array}$ & $\begin{array}{l}\text { Context } \\
\text { relevance } \\
\text { CR(r) }\end{array}$ & $\begin{array}{c}\text { Non-spatial } \\
\text { effect } \\
\text { function } \\
E(a, n)\end{array}$ & $\begin{array}{l}\text { Marginal } \\
\text { context } \\
\text { effect (on } \\
\text { circle line r) }\end{array}$ & $\begin{array}{l}\text { Context } \\
\text { effect within } \\
\text { circle(r) }\end{array}$ \\
\hline A & $\begin{array}{l}\text { Simple } \\
\text { distance } \\
\text { decay }\end{array}$ & $\begin{array}{c}\text { equal } \\
\text { distribution } \\
n(r)=\text { const. }\end{array}$ & & & & & & & & \\
\hline & $\begin{array}{c}1 / r \\
(r>0)\end{array}$ & n 2 r $\pi$ & & & & & & & & \\
\hline i & $\hat{i}$ & $\uparrow$ & $\uparrow$ & $\uparrow$ & $\uparrow$ & $\uparrow$ & $\uparrow$ & linear & $\uparrow$ & $\uparrow$ \\
\hline ii & & & & & & & & $\begin{array}{l}\text { decreasing } \\
\text { returns: } \\
\text { sqrt }(X)\end{array}$ & $\uparrow$ & $\downarrow$ \\
\hline & & $\begin{array}{l}\text { distribution } \\
w / \text { clustering } \\
n(r)=\text { const./r }\end{array}$ & & & & & & & & \\
\hline iii & & $\uparrow$ & $\uparrow$ & $\uparrow$ & $\uparrow$ & & & linear & $\uparrow$ & $\uparrow$ \\
\hline iv & & & & & & & & $\begin{array}{l}\text { decreasing } \\
\text { returns: } \\
\text { sqrt }(X)\end{array}$ & & \\
\hline B & $\begin{array}{l}\text { Gravity } \\
\text { model }\end{array}$ & $\begin{array}{c}\text { equal } \\
\text { distribution } \\
n(r)=\text { const. }\end{array}$ & & & & & & & & \\
\hline & $\begin{array}{l}1 / r^{2} \\
(r>0)\end{array}$ & n 2 r $\pi$ & & & & & & & & \\
\hline $\mathbf{i}$ & $\uparrow$ & $\uparrow$ & $\uparrow$ & $\uparrow$ & $\uparrow$ & & $\uparrow$ & linear & $\uparrow$ & $\uparrow$ \\
\hline ii & & & & & & & & $\begin{array}{l}\text { decreasing } \\
\text { returns: } \\
\text { sqrt }(X)\end{array}$ & & \\
\hline & & $\begin{array}{l}\text { distribution } \\
w / \text { clustering } \\
n(r)=\text { const./r }\end{array}$ & & & & & & & & \\
\hline iii & & $\uparrow$ & $\uparrow$ & $\uparrow$ & $\uparrow$ & $\uparrow$ & $\uparrow$ & linear & $\uparrow$ & $\uparrow$ \\
\hline iv & & & & & & & & $\begin{array}{l}\text { decreasing } \\
\text { returns: } \\
\operatorname{sqrt}(X)\end{array}$ & & \\
\hline C & $\begin{array}{l}\text { Normal } \\
\text { density }\end{array}$ & $\begin{array}{c}\text { equal } \\
\text { distribution } \\
n(r)=\text { const. }\end{array}$ & & & & & & & & \\
\hline & a ND $(r \mid 0 ; b)$ & $\mathrm{n} 2 \mathrm{r} \pi$ & & & & & & & & \\
\hline $\mathbf{i}$ & $\uparrow$ & $\uparrow$ & 4 & $\uparrow$ & $\uparrow$ & & $\uparrow$ & linear & $\uparrow$ & $\uparrow$ \\
\hline ii & & & & & & & & $\begin{array}{l}\text { decreasing } \\
\text { returns: } \\
\text { sqrt }(X)\end{array}$ & & \\
\hline & & $\begin{array}{l}\text { distribution } \\
w / \text { clustering } \\
n(r)=\text { const./r }\end{array}$ & & & & & & & & \\
\hline iii & & $\uparrow$ & & $\uparrow$ & $\uparrow$ & & & linear & $\hat{A}$ & \\
\hline iv & & & & & & & & $\begin{array}{l}\text { decreasing } \\
\text { returns: } \\
\text { sqrt }(X)\end{array}$ & & \\
\hline
\end{tabular}

Fig 6. Analytical examples: selection of possible distributions and related outcomes

\section{(schematic figures)—two-dimensional space}

All X-axes represent distance/radius $\mathrm{r}$. 
Radius-based illustrations of applications in three-dimensional space are displayed in Fig 7. In example (A), simple distance decay is again assumed (i.e., the relevance of context is proportional to the inverse of distance) (11). Context elements are assumed to be equally distributed within the three-dimensional space (12). From these assumptions and the formulae developed above, the marginal context relevance (13), the area-based context relevance (17), and all the intermediate steps can be calculated. Depending on the respective combination function (18), the distance-based effect pattern of both the marginal context effect (19) and the context effect within the area (20) can be derived.

In models (i) and (ii), these effects increase with distance, regardless of whether a linear combination function or a function representing decreasing returns (square root of context relevance) is chosen. This result is unrealistic because this would mean measuring larger and larger context effects with increasing sizes of the context. Models (iii) and (iv) consider local clustering of context elements around the objects of study (agglomeration). In the case of a clustering factor (1/r), the resulting context effects can be assumed to be constant with distance.

Example $(B)$ follows the original form of the gravity model, assuming a decline of the element-specific relevance that is proportional to $1 / \mathrm{r}^{2}$. For both alternatives, an equal distribution of context elements and central clustering, the expected patterns of (area-based) context effects decline with distance, to varying degrees. In example $(C)$, it is assumed that the element-specific relevance follows a normal density. In this case, we can again expect non-monotonic patterns of context effects (i.e., that there is a maximum of expected context effects at a certain distance from the object of study). 


\begin{tabular}{|c|c|c|c|c|c|c|c|c|c|c|}
\hline & (11) & (12) & (13) & (14) & (15) & (16) & (17) & (18) & (19) & (20) \\
\hline & $\begin{array}{c}\text { Relevance } \\
\text { of opp. by } \\
\text { distance: } \\
\text { R(r) }\end{array}$ & $\begin{array}{c}\text { Number of } \\
\text { opp. by } \\
\text { distance: } \\
N(r)\end{array}$ & $\begin{array}{l}\text { Marginal } \\
\text { relevance } \\
\text { (on ball } \\
\text { surface r) }\end{array}$ & $\begin{array}{l}\text { No. of opp } \\
\text { within ball } \\
\text { (r) }\end{array}$ & $\begin{array}{c}\text { Average } \\
\text { relevance } \\
\text { within ball } \\
(r)\end{array}$ & $\begin{array}{l}\text { Coverage } \\
\text { within ball } \\
\text { (r) }\end{array}$ & $\begin{array}{c}\text { Context } \\
\text { relevance } \\
\text { CR(r) }\end{array}$ & $\begin{array}{c}\text { Non-spatial } \\
\text { effect } \\
\text { function } \\
E(a, n) \\
\end{array}$ & $\begin{array}{c}\text { Marginal } \\
\text { context } \\
\text { effect (on } \\
\text { ball surface) }\end{array}$ & $\begin{array}{l}\text { Context } \\
\text { effect within } \\
\text { ball }(r)\end{array}$ \\
\hline A & $\begin{array}{c}\text { Simple } \\
\text { distance } \\
\text { decay }\end{array}$ & $\begin{array}{c}\text { equal } \\
\text { distribution } \\
\mathrm{n}(\mathrm{r})=\text { const. }\end{array}$ & & & & & & & & \\
\hline & $\begin{array}{c}1 / r \\
(r>0)\end{array}$ & $\mathrm{n} 4 \mathrm{r}^{2} \pi$ & & & & & & & & \\
\hline $\mathbf{i}$ & $\uparrow$ & $\uparrow$ & $\uparrow$ & $\uparrow$ & $\uparrow$ & $\uparrow$ & $\uparrow$ & linear & $\uparrow$ & $\uparrow$ \\
\hline ii & & & & & & & & $\begin{array}{l}\text { decreasing } \\
\text { returns: } \\
\text { sqrt }(X)\end{array}$ & & \\
\hline & & $\begin{array}{l}\text { distribution } \\
\mathrm{w} / \text { clustering } \\
\mathrm{n}(\mathrm{r})=\text { const. } / \mathrm{r}\end{array}$ & & & & & & & & \\
\hline iii & & $\uparrow$ & $\uparrow$ & $\uparrow$ & $\uparrow$ & $\uparrow$ & $\uparrow$ & linear & $\uparrow$ & $\uparrow$ \\
\hline iv & & & & & & & & $\begin{array}{l}\text { decreasing } \\
\text { returns: } \\
\text { sqrt }(X)\end{array}$ & $\uparrow$ & † \\
\hline B & $\begin{array}{l}\text { Gravity } \\
\text { model }\end{array}$ & $\begin{array}{c}\text { equal } \\
\text { distribution } \\
n(r)=\text { const. }\end{array}$ & & & & & & & & \\
\hline & $\begin{array}{l}1 / r^{2} \\
(r>0)\end{array}$ & $\mathrm{n} 4 \mathrm{r}^{2} \pi$ & & & & & & & & \\
\hline $\mathbf{i}$ & $\uparrow$ & $\uparrow$ & $\uparrow$ & $\uparrow$ & $\uparrow$ & $\uparrow$ & & linear & $\uparrow$ & \\
\hline ii & & & & & & & & $\begin{array}{l}\text { decreasing } \\
\text { returns: } \\
\operatorname{sqrt}(X)\end{array}$ & $\uparrow$ & \\
\hline & & $\begin{array}{l}\text { distribution } \\
w / \text { clustering } \\
n(r)=\text { const. } / r\end{array}$ & & & & & & & & \\
\hline iii & & $\uparrow$ & & $\uparrow$ & $\uparrow$ & & $\hat{\imath}$ & linear & $\uparrow$ & $\uparrow$ \\
\hline iv & & & & & & & & $\begin{array}{l}\text { decreasing } \\
\text { returns: } \\
\text { sqrt }(X)\end{array}$ & & \\
\hline C & $\begin{array}{l}\text { Normal } \\
\text { density }\end{array}$ & $\begin{array}{c}\text { equal } \\
\text { distribution } \\
\mathrm{n}(\mathrm{r})=\text { const. }\end{array}$ & & & & & & & & \\
\hline & a ND $(r \mid 0 ; b)$ & $n 4 r^{2} \pi$ & & & & & & & & \\
\hline $\mathbf{i}$ & $\uparrow$ & $\uparrow$ & & $\uparrow$ & 4 & A & & linear & & \\
\hline ii & & & & & & & & $\begin{array}{l}\text { decreasing } \\
\text { returns: } \\
\text { sqrt }(X)\end{array}$ & & \\
\hline & & $\begin{array}{l}\text { distribution } \\
w / \text { clustering } \\
n(r)=\text { const. } / r\end{array}$ & & & & & & & & \\
\hline iii & & $\uparrow$ & & $\uparrow$ & & & & linear & & \\
\hline iv & & & & & & & & $\begin{array}{l}\text { decreasing } \\
\text { returns: } \\
\text { sqrt }(X)\end{array}$ & & \\
\hline
\end{tabular}

Fig 7. Analytical examples: selection of possible distributions and related outcomes

(schematic figures)—three-dimensional space

All X-axes represent distance/radius $\mathrm{r}$. 


\section{Conclusions}

Theoretical considerations or empirical information about entity-specific spatial relationships alone (how important an object is in distance r) are typically not sufficient to determine the space of relevant context effects. In this sense, simple gravity models or similar models are not a fully adequate or sufficient basis for a (hierarchical) spatial context analysis. In addition to specific assumptions about the element-specific relevance, the distribution of context elements and a combination function need to be specified. If this is done, however, expected effect patterns can be derived relatively easily. In this way, it becomes possible to understand why non-monotonic patterns in scale-related context effects have been repeatedly found (see $[30,31]$ ) in spite of common assumptions of distance decay in relevance.

In many cases, a distance-related maximum of the context relevance can be expected (i.e., there is a range where the expected context effect is maximal), so that an "optimal” range for the measurement of context effects can be specified. In others, a steady decline with distance in the magnitude of context effects can be expected. At least in limited ranges, also constant effects can be plausible. Finally, it is important to distinguish between applications in two- and three-dimensional space and between marginal and area- or volume-based contexts. Different research questions will require different substantive assumptions and, hence, lead to specific results, but this general model may help to distinguish between different definitions of context relevance and to specify the steps necessary for the calculation of spatial context patterns. The model may also substantiate implications of the modifiable areal unit problem and help to identify unrealistic assumptions. Of course, different context attributes may have different influences, and in applications, it may be an additional challenge to determine the combined effect in the presence of different context influences. 


\section{Acknowledgments}

Part of this paper was written while the author was visiting at Nuffield College, University of Oxford. I would like to thank, in particular, Francesco Billari for his hospitality and also David Cox for some helpful conversations.

\section{References}

1. Newton I. Philosophiae naturalis principia mathematica. London: Joseph Streater; 1687.

2. Tobler WR. A computer movie simulating urban growth in the Detroit region. Econ Geogr. 1970;46: 234-40.

3. Taylor PJ. Distance decay in spatial interactions. Norwich, UK: Geo Books; 1975.

4. Zipf, GK. Human behaviour and the principle of least effort: an introduction to human ecology. Menlo Park, CA: Addison Wesley; 1949.

5. Östh J, Clark WA, Malmberg, B. Measuring the scale of segregation using k-nearest neighbor aggregates. Geogr Anal. 2015;47: 34-49.

6. Bronfenbrenner $U$. The ecology of human development: experiments by nature and design. Cambridge, MA: Harvard University Press; 1979.

7. Kareiva P. Population dynamics in spatially complex environments: theory and data. Philos Trans R Soc Lond B Biol Sci. 1990;330: 175-90.

8. Hubbell SP. The unified neutral theory of biodiversity and biogeography. Princeton, NJ: Princeton University Press; 2001.

9. Shebilske WL. Context effects and efferent factors in perception and cognition. In: Prinz W, Sanders AF, editors. Cognition and Motor Processes. Berlin, Germany: Springer; 1984, p. 99-119. 
10. Hipp JR, Boessen A. Egohoods as waves washing across the city: a new measure of “neighborhoods.” Criminology. 2013;51: 287-327.

11. Oliver LN, Schurman N, Hall AW. Comparing circular and network buffers to examine the influence of land use on walking for leisure and errands. Int J Health Geogr. 2007;6: $41-51$

12. Wiens JA. Spatial scaling in ecology. Funct Ecol. 1989;3: 385-97.

13. Levin, SA. The problem of pattern and scale in ecology. Ecology. 1992;73: 1943-67.

14. Chave, J. The problem of pattern and scale in ecology: what have we learned in 20 years? Ecol Lett. 2013;16: 4-16.

15. Bader MD, Ailshire JA. Creating measures of theoretically relevant neighbourhood attributes at multiple spatial scales. Sociol Methodol. 2014;44: 322-68.

16. Roland J, Taylor PD. Insect parasitoid species respond to forest structure at different spatial scales. Nature. 1997;386: 710-13.

17. Andersson R, Musterd S. What scale matters? Exploring the relationships between individuals' social position, neighbourhood context and the scale of neighbourhood. Geogr Ann Ser B. 2010;92: 23-43.

18. Nekola JC, White PS. The distance decay of similarity in biogeography and ecology. J Biogeogr. 1999;26: 867-78.

19. Gehlke CE, Biehl K. Certain effects of grouping upon the size of the correlation coefficient in census tract material. J Am Stat Assoc. 1934;29: 169-70.

20. Openshaw S. The modifiable areal unit problem. Norwich, UK: Geo Books; 1983.

21. Wong D. The modifiable areal unit problem (MAUP). In: Fotheringham AS., Rogerson P, editors. The SAGE handbook of spatial analysis. Los Angeles, CA: Sage; 2009, p. 10524.

22. Tinbergen J. Shaping the world economy: suggestions for an international economic policy. New York, NY: The Twentieth Century Fund; 1962. 
23. Hua V, Porell F. A critical review of the development of the gravity model. Int Reg Sci Rev. 1979;4: 97-126.

24. Haynes K, Fotheringham AS. Gravity and spatial interaction models. Beverly Hills, CA: Sage; 1984.

25. Stouffer SA. Intervening opportunities: a theory relating mobility and distance. Am Sociol Rev. 1940;5: 845-67.

26. Stewart JQ. An inverse distance variation for certain social influences. Science. 1941;93: 89-90.

27. Latané B, Liu JH, Nowak A, Bonevento M, Zheng L. Distance matters: physical space and social impact. Pers Soc Psychol Bull. 1995;21: 795-805.

28. Martínez LM, Viegas JM. A new approach to modelling distance-decay functions for accessibility assessment in transport studies. J Transp Geogr. 2013;26: 87-96.

29. Halás M, Klapka P, Kladivo P. Distance-decay functions for daily travel-to-work flows. J Transp Geogr. 2014;35: 107-19.

30. Thies C, Steffan-Dewenter I, Tscharntke T. Effects of landscape context on herbivory and parasitism at different spatial scales. Oikos. 2003;101: 18-25.

31. Avelino J, Romero-Gurdián A, Cruz-Cuellar HF, Declerck FA. Landscape context and scale differentially impact coffee leaf rust, coffee berry borer, and coffee root-knot nematodes. Ecol Appl. 2012;22: 584-96. 